\title{
Evolution of the human menopause
}

\author{
Daryl P. Shanley and Thomas B.L. Kirkwood*
}

\begin{abstract}
Summary
Menopause is an evolutionary puzzle since an early end to reproduction seems contrary to maximising Darwinian fitness. Several theories have been proposed to explain why menopause might have evolved, all based on unusual aspects of the human life history. One theory is that menopause follows from the extreme altriciality of human babies, coupled with the difficulty in giving birth due to the large neonatal brain size and the growing risk of child-bearing at older ages. There may be little advantage for an older mother in running the increased risk of a further pregnancy when existing offspring depend critically on her survival. An alternative theory is that within kin groups menopause enhances fitness by producing post-reproductive grandmothers who can assist their adult daughters. Such theories need careful quantitative assessment to see whether the fitness benefits are sufficient to outweigh the costs, particularly in circumstances of relatively high background mortality typical of ancestral environments. We show that individual theories fail this test, but that a combined model incorporating both hypotheses can explain why menopause may have evolved. BioEssays 23:282-287, 2001. (c) 2001 John Wiley \& Sons, Inc.
\end{abstract}

\section{Introduction}

Despite half a century of enquiry, the human menopause remains an evolutionary puzzle. ${ }^{(1)}$ Fertility loss in women (menopause) occurs at a remarkably similar age-around 50 years - in all human populations and, when compared with other species, happens unusually early in the life span. The proximate cause of reproductive senescence in mammalian females is the exhaustion of ovarian oocytes, accompanied by degenerative changes in reproduction-associated elements of the neuroendocrine system. ${ }^{(5)}$ While oocyte depletion is the trigger for menopause, however, there is no clear reason why the size of the initial oocyte stock should not have increased in line with human longevity. Equally, although the increase in chromosome abnormalities with maternal age points to an age-related decline in oocyte quality, which might itself offer a reason to cease reproduction, there is no compelling reason why a longer "shelf-life" could not have evolved through better

Department of Gerontology, University of Newcastle, UK.

Funding agency: The Wellcome Trust

${ }^{*}$ Correspondence to: Professor Thomas Kirkwood, Department of Gerontology, University of Newcastle, Institute for the Health of the Elderly, Newcastle General Hospital, Westgate Road, Newcastle upon Tyne NE4 6BE. E-mail: Tom.Kirkwood@ncl.ac.uk cellular repair (such as probably explains the extended functional longevity of human neurons). It is thus appropriate to ask if the ultimate reason for the existence of menopause lies in the evolutionary biology of the human life history.

The two major evolutionary hypotheses to explain menopause are founded on the extreme altriciality of human infants and the extensive opportunities for intergenerational cooperation within kin groups. ${ }^{(6,7)}$ The altriciality of human offspring appears to be the result of a compromise driven by the evolution of an increasingly large brain in the hominid ancestral lineage and the pelvic constraint on the birth canal. On the one hand, the human neonatal brain size is near to the limit that is compatible with safe delivery, and even so presents considerable mortality risk to the mother in cases of birth complications. On the other hand, the newborn human infant still requires its brain to grow and develop for a considerable period before it is capable of any kind of independent existence, which renders it highly dependent on adult (usually maternal) attention for its survival. Given that maternal mortality increases with age and that maternal death will seriously compromise the survival of any existing dependent offspring, it appears to make sense to cease having more children when the risks outweigh the benefits. Nevertheless, Homo sapiens is unique in the extent to which kin assist in care and provisioning of young. ${ }^{(8,9)}$ Orphans regularly do survive even in adverse environments. Thus, an alternative theory is that menopause enhances fitness by producing post-reproductive grandmothers who can assist their adult offspring by sharing in the burden of provisioning and protecting their grandchildren. Previous attempts to model these ideas have suggested that the assistance provided by grandmothers would have to be unrealistically high to make the necessary difference. ${ }^{(10-13)}$ There has as yet been no attempt to evaluate these alternative hypotheses on a comparative basis, however, nor has there been any assessment of whether the various elements that might provide an evolutionary explanation of menopause might in some way complement each other. In this paper we describe such an approach.

\section{How we analysed the data}

We have analysed life table data from a Taiwanese pastoral population of $1906^{(14)}$ (Fig. 1a). These data are typical of those recorded in other traditional (e.g. hunter-gatherer) populations and have previously been used for studies on evolution of senescence. ${ }^{(14)}$ If the menopause is an evolved human trait, its origins may best be revealed under these conditions. Our 


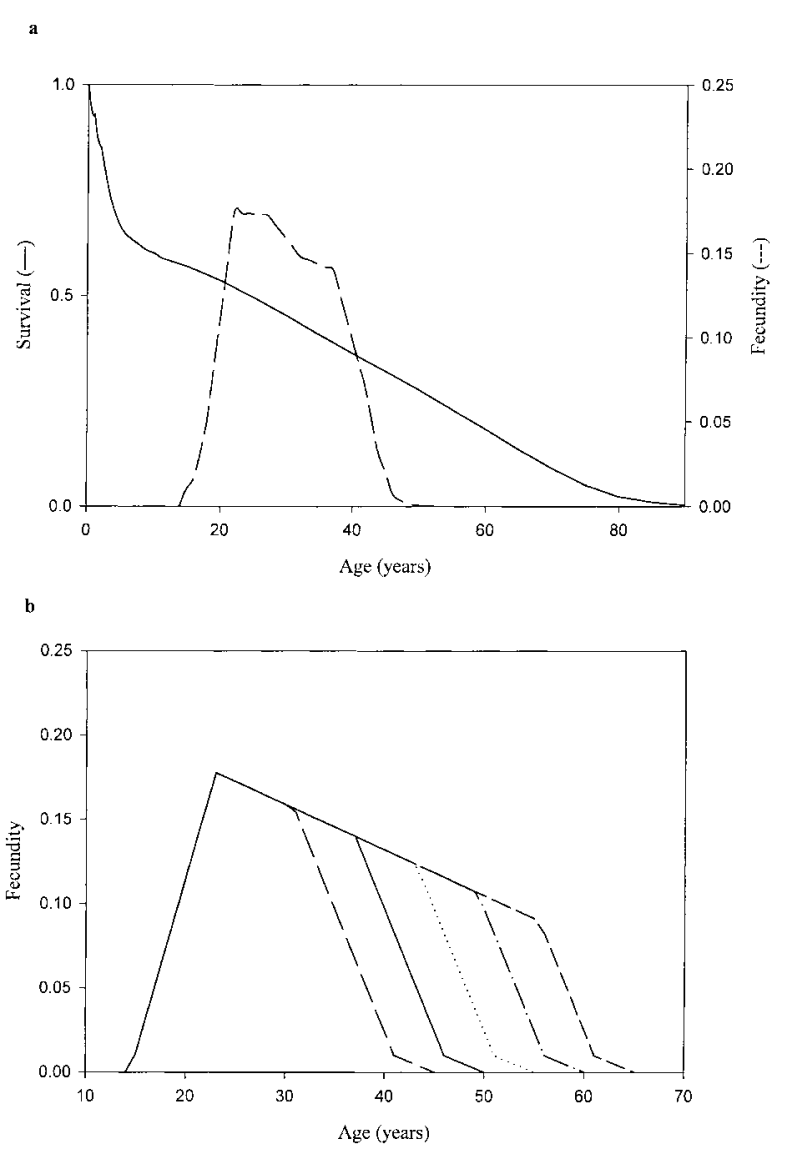

Figure 1. a: The variation of survival and fecundity with age of a Taiwanese human population in $1906 .^{(14)}$ b: A geometrical representation of the fecundity data, and hypothetical fecundity with ages of menopause in the range of 45-65 years.

model (see Appendix) groups the female population into different classes with respect to life history state. The model is based on the schedules of adult and juvenile mortality and fertility. Total annual mortality at age $x$, which includes a contribution from background senescence, is partitioned into separate components $\mu_{i}(\boldsymbol{x})$ associated with specific features, such as childbearing. The probability $\phi_{i}(x)$ of being exposed to individual components of mortality is also calculated as a function of age. The predicted survival and fecundity curves are obtained and the free parameters of the model estimated by least-squares fit of the predicted survival and fecundity to the raw data (Fig. 1a). Each class experiences reproduction and mortality appropriate to its state, which may be affected by the giving or receiving of intergenerational support. For infants, there are four possible states defined by the presence or absence of mother and grandmother. Young adults have high fertility and are exposed to mortality risks linked with childbirth and childcare, which may be reduced by assistance of a grandmother. Middle-aged adults experience declining fertility and an age-related increase in the risk of childbirth. Older adults show a senescence-related increase in mortality (independent of reproduction) and those with no children of their own may support their grandchildren. The probability of belonging in each state class is calculated from the survival and fertility data. Predicted survival $I_{\text {calc }}$ at age $x+1$ is calculated by exposing the cohort of survivors at age $x$ to components of mortality $\mu_{i}$ with probability $\phi_{i}$ as follows:

$$
I_{\text {calc }}(x+1)=I_{\text {calc }}(x) \Sigma \Phi(x) \mathrm{e}^{-\Sigma \mu(x)},
$$

where $\Phi(x)$ is the probability of being in any given state as a function of the product of the probabilities $\phi_{i}(x)$ and $\left(1-\phi_{i}(x)\right)$, and $\Sigma \mu(x)$ is the total mortality associated with that state.

These data are then used to compute the intrinsic rate of natural increase, $r$, which we take as a measure of evolutionary fitness. By varying the fertility schedule as shown in Fig. 1b, and by using the mortality and fecundity costs and benefits determined from the data and model, we explore how selection might influence the age at which fertility ceases. Since the form assumed in Fig. $1 \mathrm{~b}$ was to some extent arbitrary, we also used other forms to vary age at menopause with qualitatively similar results; these are not shown here but are available on request.

If for a given model there is a fitness optimum (a peak in the fitness curve) associated with ceasing reproduction early, this indicates that the hypothesis on which the model is based can explain evolution of menopause. If there is no optimum (i.e. if $r$ continues to increase as fertility is extended to later ages), this suggests that the model cannot account for evolution of menopause.

\section{What the analysis tells us}

\section{Model 1: altriciality of human infants}

We consider first whether the dependency of human infants combined with the increased mortality risk of reproducing at later ages can explain menopause, in the absence of any assistance from grandmothers. This is done primarily by increasing the exponential rate parameter, $\beta_{\text {birth }}$, while at the same time adjusting the constant parameters, $\alpha_{\text {birth }}$ and $\mu_{\text {child }}\left(x_{\text {maturity }}\right)$, to meet the constraint that the model fits the data. Fig. 2a shows that even very high levels of childbirthassociated mortality are insufficient to offset the benefit of reproducing to later ages, even though the latter benefit may be slight. Fig. $2 b$ shows the additional effects, for the "medium" case of Fig. 2a, of assuming increased child mortality if a mother dies. The impact of the mother's death on juvenile mortality is assumed to vary with age, so that a child younger than 2 years cannot survive, whereas a child of 15 years is unaffected. The examples show cases of $5-$ and 20-fold higher mortality at age 5 compared to children with surviving mothers. There is some evolutionary support for 


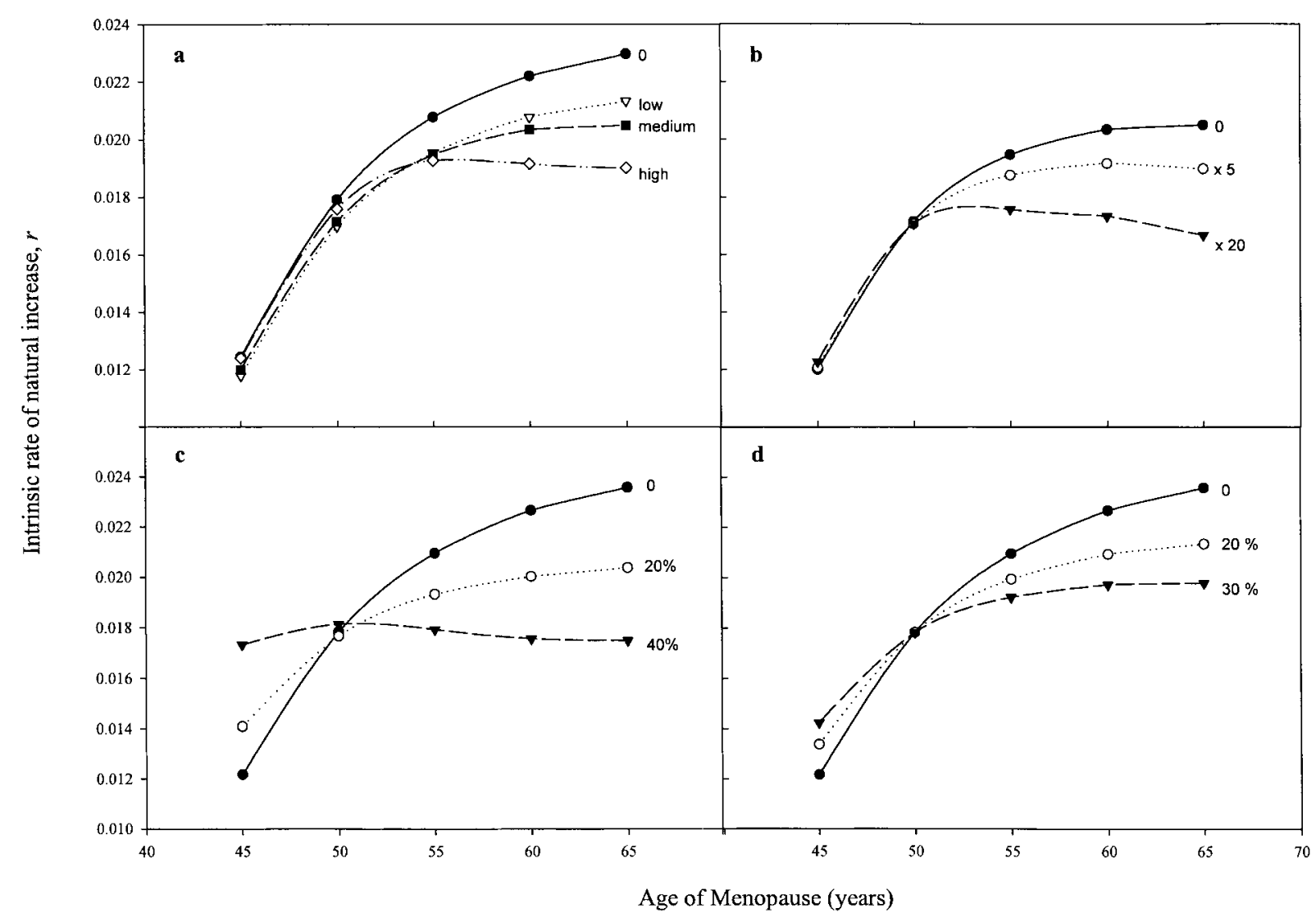

Figure 2. Relationships between the intrinsic rate of natural increase and age at menopause for models representing individual features. a: Results for four levels of age-related increase in maternal mortality in childbirth (parameter values: $\mu_{\text {depgrand }}, \alpha_{\text {birth }}$, $\beta_{\text {birth }}=0.019,0,0$ or $0.014,0.00293,0.1$ or $0.015,0.000485,0.181$ or $0.017,0.0000001,0.5$, respectively, and in all cases $\mu_{\text {child }}=0.012$, $\left.\mu_{\text {assist }}=-0.01\right)$. b: Three levels for the effect of the mother's death on juvenile survival giving a mortality differential at age 5 of 0,5 and 20 (parameter values: $(\alpha, \beta)_{\text {livingmother, }}(\alpha, \beta)_{\text {orphan }}=(0,0),(0,0)$ or $(0.226,1.074),(1.107,1.78)$ or $(0.118,0.84),(10.745,2.666)$, respectively, and in all cases $\left.\mu_{\text {child }}=0.012, \mu_{\text {assist }}=-0.01, \mu_{\text {depgrand }}=0.015, \alpha_{\text {birth }}=0.000485, \beta_{\text {birth }}=0.181\right)$. c: Three levels of fertility enhancement due to the mothers assistance (parameter values: $\alpha_{b}=0$ or 0.24 or 0.48 , respectively, and in all cases $\left.\mu_{\text {child }}=0.0078, \mu_{\text {assist }}=0, \mu_{\text {depgrand }}=0.02\right)$. d: Three levels of reduction in the mortality of children $(<15$ years $)$ due to a grandmother's care ( parameter values: $\alpha_{\mu}=0$ or -0.0335 or -0.0574 , respectively, and in all cases $\mu_{\text {child }}=0.0078, \mu_{\text {assist }}=0, \mu_{\text {depgrand }}=0.02$ ). All results use $\mu_{\text {adult }}=0.01$, and $\mu_{0}=0.0005$ and $\beta=0.085$ as the parameters of the standard Gompertz-Makeham description of agerelated mortality. ${ }^{(23)}$

menopause in the case of an extreme 20-fold increase in mortality of orphans, but not for the more realistic 5-fold increase. ${ }^{(11)}$ Increased orphan mortality is insufficient on its own, i.e. without an age-related increase in maternal mortality, to explain menopause even with the draconian assumption of a 20 -fold increase (case not shown).

\section{Model 2: intergenerational assistance}

We consider next the impact of a post-reproductive woman on her daughter's reproductive success, in the absence of increased mortality risk of reproducing at later ages. Intergenerational help might increase the adult daughter's fertility, e.g. by freeing her from some of the energy burdens imposed by existing children, reduce her mortality in childbirth, or reduce grandchild mortality. Food provision is an important determinant of adult fertility. ${ }^{(15)}$ The presence of a grandmother can enhance juvenile growth ${ }^{(8)}$ and has been shown to reduce mortality substantially. ${ }^{(16)}$ Fig. $2 c$ shows the effects of assuming that help increases an adult daughter's fertility. Overall enhancement levels of $20 \%$ and $40 \%$ are shown, menopause becoming adaptive only at very high levels of fertility enhancement, i.e. above $40 \%$. Bearing in mind that only a fraction of women will benefit from enhancement, the actual level of benefit received in the model by these women is substantially greater; such high levels of fertility enhancement are unlikely. Fig. $2 d$ shows the effect of assuming a grand-

\section{BioEssays 23.3}




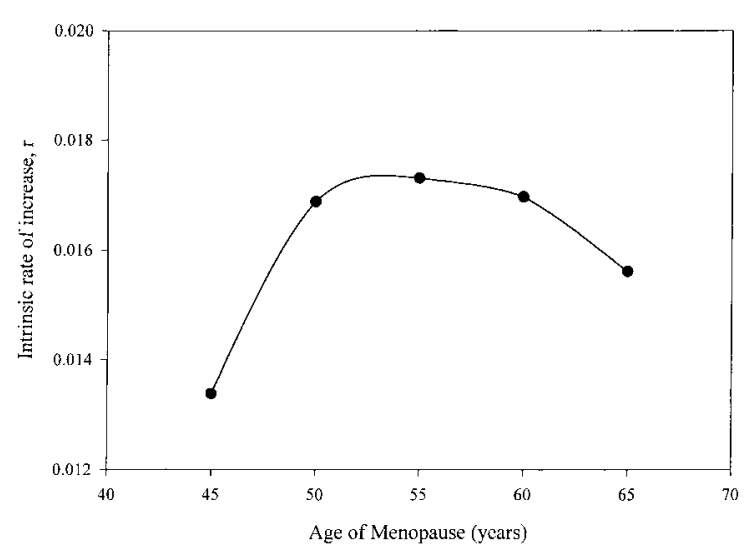

Figure 3. Relationship between the intrinsic rate of natural increase and age at menopause for a composite model where maternal mortality increases moderately with age, $10 \%$ of fertility is attributable to maternal assistance, juvenile survival is 5 -fold higher with a living mother and child mortality is reduced by $10 \%$ with grandmaternal care. (Parameter values: $\mu_{\text {child }}=0.012, \quad \mu_{a_{\text {ssist }}}=-0.01, \quad \mu_{\text {depgrand }}=0.015,(\alpha, \beta)_{\text {birth }}=$ $(0.000485,0.181), \quad \alpha_{b}=0.12, \quad(\alpha, \beta)_{\text {livingmother }}=(0.226,1.074)$, $(\alpha, \beta)_{\text {orphan }}=(1.107,1.78)$ and $\alpha_{\mu}=-0.01488$.

mother increases juvenile survival. Even in the case that a grandmother reduces mortality by $30 \%$, there is no apparent selection for menopause.

\section{Composite model}

The previous analyses show that neither of the individual hypotheses can account for evolution of menopause on its own, but a very different picture emerges if we combine the factors from the two models considered above in a composite model (Fig. 3). Here we assume an age-dependence of maternal mortality that corresponds to the medium case of Fig. 2a, a 5-fold effect of maternal death on child mortality at age 5 , a $10 \%$ mortality reduction for grandchildren, and a $10 \%$ fertility enhancement due to presence of post-reproductive mother. These contributions are compatible with the available data and indicate support for an adaptive role of menopause.

Although the composite model contains a fairly large number of terms, these are either estimated from data or tightly constrained by the fitting process, indicating that our conclusions have general validity. To address issues of robustness and sensitivity of the model predictions to varying our specific assumptions, we carried out a series of analyses in which different combinations of parameter values were assessed for their impact on our results. The general pattern presented above was found to be very robust, and the sensitivity analysis also allowed us to assign a ranking to the importance of each factor in the combined model as follows (most important first): age-dependence of maternal mor- tality $>$ daughter's fertility enhancement $>$ reduced grandchild mortality $>$ orphan mortality $>$ reduced daughter's maternal mortality. The primacy of the age-dependance of maternal mortality can be readily appreciated when we note that this factor affects not only the survival of the older females themselves but also their potential for making further contributions to child and grandchild survival and to the fecundity of their adult daughters.

\section{Conclusions and further discussion}

Our finding that a composite model readily explains menopause, while individual factors do not, can account for the uniqueness of the human life history. Other social species, such as baboons and lions, show intergenerational exchange but menopause has not evolved. ${ }^{(17)}$ A key difference may be the high risk of mortality in human childbirth, probably due to the large neonatal head size and constraints on the pelvis imposed by bipedal gait. Records of American females show an exponential increase in risk of maternal mortality with age. ${ }^{(18)}$ This is also seen in captive rhesus macaques, which unlike other apes, share the human problem of a difficult birth $^{(19)}$ and are reported to have an early termination of fertility. ${ }^{(3)}$ However, our model shows that increasing maternal mortality in childbirth does not, on its own, eliminate the fitness benefit that older females receive through their own reproduction. Older human females enhance the survival of grandchildren through protection or provisioning and they lift some of the burden from adult daughters, enabling higher levels of fertility and an earlier age of weaning. ${ }^{(20)}$ In lions, grandmothers may assist by provisioning their daughter's progeny but they do this through lactation, which requires that they remain reproductive themselves.

Another important difference between humans and other species concerns the relative scaling of key events in the life history. The age at which a female becomes mature, relative to life span and her age at reproductive senescence, affects the probability of her receiving assistance in young adulthood. Humans have an unusually long maturation period so mothers are relatively old before their daughters are mature. Female baboons mature at 3 years and have a potential life span of nearly 30 years. If a young adult baboon were to have the same chance of receiving assistance from a post-reproductive mother as seen in the Taiwanese data, fertility would have to cease at 10 years of age.

Our model suggests that evolution of menopause is a process that has its roots in complex features of the human life history, including the unusual importance of the welldocumented kin interactions. The results of the model clearly show that the complex interplay between the key parameters affecting different periods of the life history are not the same as the sum of their independent actions. From a biomathematical perspective, this is not surprising given that the model population converges iteratively to an equilibrium state and 
incorporates feedback mechanisms characteristic of the interconnected nature of life history variables.

We note that even for the composite model the fitness peak is not particularly sharp and spreads above the observed age of menopause, which is around 50 . This is most likely due to the absence from our model of additional factors that would be required fully to represent the human life history. For example, we do not explicitly include the physiological costs of the extra life-long somatic maintenance that might be required to preserve reproductive viability to later ages; these costs would be likely to require the diversion of metabolic resources from earlier reproductive effort, as suggested by the disposable soma theory of ageing. ${ }^{(21)}$ Another example of a factor that we have not explicitly considered but which will reinforce the adaptive role of menopause is infanticide. ${ }^{(22)}$ Infanticide is common in humans and may serve to protect mothers in circumstances when the burden of a young child threatens her own survival. Infanticide is particularly common when a female loses a partner and attracts the attention of other males. The risk of infanticide will add to the evolutionary advantage of menopause already identified in our analysis and would help to sharpen the fitness peak.

\section{Appendix}

\section{Adult mortality}

Adult mortality is partitioned into four components. The first is the standard Gompertz-Makeham description of age-related mortality ${ }^{(23)}$ with probability of exposure of one, comprising a constant for age-independent extrinsic causes of death, $\mu_{\text {adult, }}$ plus a senescence-related term, $\mu_{\text {senescence }}(\boldsymbol{x})=\mu_{0}$ $\mathrm{e}^{\beta\left(x-x_{\text {maturity }}\right.}$. The second is a mortality cost of reproduction, $\mu_{\text {child }}(x)$, combining mortality in childbirth and a mortality cost of caring for dependent children ( $0-3$ years). The probabilities of exposure to these are $\phi_{\text {birth }}(x)=2 b_{x}$ and $\phi_{\text {depchild }}(x)=$ $2\left(b_{x}+l_{1} b_{x-1}+l_{2} b_{x-2}\right)$, respectively. Mortality in childbirth increases with age, one estimate indicating a ten-fold higher risk at age 40 years than at 20 years ${ }^{(18)}$. A two-component model is assumed, $\mu_{\text {child }}(\boldsymbol{x})=\left(\mu_{\text {child }}\left(x_{\text {maturity }}\right)-\alpha_{\text {birth }}\right)+\alpha_{\text {birth }}$ $\mathrm{e}^{\beta_{\text {birth }}\left(x-x_{\text {maturity }}\right)}$, similar to the above model for background mortality. The third component is a mortality benefit (negative cost) to a mother who receives assistance from her mother with the care of dependent infants, $\mu_{\text {assist }}$. The probability that an adult female (age $x$ ) has a living mother (age $y$ ) who does not have dependent children of her own is

$$
\phi_{\text {assist }}(x)=\frac{1}{\sum b_{z} I_{z}} \sum_{y=x+x_{\text {maturity }}}^{x+x_{\text {menopause }}} b_{y-x} l_{y}\left(1-\phi_{\text {depchild }}(y)\right) \text {, }
$$

where $x_{\text {maturity }}$ and $x_{\text {menopause }}$ refer to the minimum and maximum age of reproduction. The fourth component is a mortality cost incurred by grandmothers who assist daughters with dependent grandchildren, $\mu_{\text {depgrand }}$. The probability of exposure is

$$
\phi_{\text {depgrand }}(x)=2 \sum_{z=0}^{2} I_{z} \sum_{y=x_{\text {maturity }}}^{x} b_{x-y} l_{y} b_{y-z}
$$

\section{Juvenile mortality}

The probability that a juvenile has a living mother is given by

$$
\phi_{\text {livingmother }}(\boldsymbol{x})=\frac{1}{\sum b_{z} I_{z}} \sum_{y=x_{\text {maturity }}}^{x_{\text {menopause }}} b_{y} l_{y+x} \text {. }
$$

Terms $\mu_{\text {livingmother }}(x)=\alpha_{\text {livingmother }} /(x+1)^{\beta_{\text {livingmother }} \text { and }} \mu_{\text {orphan }}$ $(x)=\alpha_{\text {orphan }} /(x-2)^{\beta_{\text {orphan }}}$, for $x>2$, describe juvenile mortality in the presence and absence of the mother. Children orphaned before 2 years are assumed always to die, and the excess mortality among orphans is thereafter assumed to diminish with age, vanishing by 15 . [Some contemporary data suggest that excess mortality of orphans reduces in an almost step-wise manner above 3 years but this is with menopause already existing and probably reflects the action of grandmaternal effects ${ }^{(8,16)}$.] The parameters are estimated by leastsquares fit to the data using the calculated probability $\phi_{\text {livingmother }}(x)$ that an infant of age $x$ has a living mother and infant survival to age $x+1$ in the form

$$
\begin{aligned}
I(x+1)= & I(x)\left(\phi_{\text {livingmother }}(x) e^{-\mu_{\text {livingmother }}(x)}\right. \\
& \left.+\left(1-\phi_{\text {livingmother }}(x)\right) e^{-\mu_{\text {orphan }}(x)}\right) .
\end{aligned}
$$

The probability that a child that was born to a mother (age $y$ ) has a living grandmother unoccupied with her own dependent children is

$$
\phi_{\text {livinggrandmother }}(\boldsymbol{x})=\frac{1}{\sum b_{z} I_{z}} \sum_{y=x_{\text {maturity }}}^{x_{\text {menopause }}} b_{y} l_{y} \phi_{\text {assist }}(y+x)
$$

Such a grandmother is assumed to reduce grandchild mortality by a fraction $\left(1-X_{\mu}\right)$ across the juvenile age range, resulting in a greater magnitude of effect at younger ages when juvenile mortality is at its most severe. Mortality, $\mu(x)$, can be written as $\mu(x)=X_{\mu} \mu_{\text {data }}(x)+\left(1-X_{\mu}\right) \mu_{\text {data }}(x)$ and the term $\left(1-X_{\mu}\right) \mu_{\text {data }}(x)$ replaced with the probability of receiving grandmaternal assistance, $\phi_{\text {livinggrandmother }}(x)$, and a constant $\alpha_{\mu}$ to give $\mu(x)=X_{\mu} \mu_{\text {data }}(x)+\phi_{\text {livinggrandmother }}(x) \alpha_{\mu}$. The constant $\alpha_{\mu}$ is estimated by least-squares fit to the data.

\section{Fecundity}

Fecundity is assumed partly dependent on maternal assistance. A fraction $X_{b}$ of the fecundity from the data is obtained by all females, the remaining fraction being gained as a result of assistance. By dividing total fecundity, $b(x)$, into the two parts, $X_{b} b_{\text {data }}(x)$ and $\left(1-X_{b}\right) b_{\text {data }}(x)$, the fraction dependent on

\section{BioEssays 23.3}


assistance is replaced with $\phi_{\text {birth }}(\boldsymbol{x}) \phi_{\text {assistance }}(\boldsymbol{x}) \alpha_{b}$ to give $b(\boldsymbol{x})=X_{b} b_{\text {data }}(\boldsymbol{x})+\phi_{\text {birth }}(\boldsymbol{x}) \phi_{\text {assistance }}(\boldsymbol{x}) \alpha_{b}$. The factor $\alpha_{b}$ is a measure of fertility enhancement through assistance, estimated by least-squares fit to the data. Using this factor and the hypothetical fecundity for females with ages of menopause other than 50 years, a similar iterative approach to that outlined above for mortality is used to determine total expected fecundity. We adjust fecundity to take account of losses due to maternal mortality in the relevant classes.

\section{References}

1. Williams GC. The Tithonus error in modern gerontology. Quart Rev Biol 1999;74:405-415

2. Gosden RG. Biology of the menopause: the causes and consequences of ovarian aging. London: Academic Press. 1985

3. Pavelka MSM, Fedigan LM. Menopause: a comparative life history perspective. Yearbook Phys Anthropol 1991;34:13-38.

4. Caro TM, Selen DW, Parish A, Frank R, Brown DM, Voland E, Mulder MB Termination of reproduction in nonhuman and human female primates. Int J Primatol 1995;16:205-220.

5. Wise PM, Krajnak KM, Kashon ML. Menopause: the aging of multiple pacemakers. Science 1996;273:67-70.

6. Williams GC. Pleiotropy, natural selection, and the evolution of senescence. Evolution 1957;11:398-411.

7. Peccei JS. The origin and evolution of menopause: the altriciality-lifespan hypothesis. Ethol Sociobiol 1995; 16:425-449.

8. Hawkes K, O'Connell JF, Blurton Jones NG. Hazda women's time allocation, offspring provisioning, and the evolution of long postmenopausal life spans. Curr Anthropol 1997;38:551-577.
9. Hawkes K, O'Connell JF, Jones NGB, Alvarez H, Charnov EL. Grandmothering, menopause, and the evolution of human life histories. Proc Natl Acad Sci USA 1998;95:1336-1339.

10. Hill K, Hurtado AM. The evolution of premature reproductive senescence and menopause in human females: an evolution of the "grandmother" hypothesis. Hum Nature 1991;2:313-350.

11. Hill K, Hurtado AM. Ache life history: the ecology and demography of a foraging people. New York: Walter de Gruyter Inc. 1996.

12. Rogers AR. Why menopause? Evol Ecol 1993;7:406-420.

13. Partridge L. Menopause for thought. Nature 1993;364:386.

14. Hamilton WD. The molding of senescence by natural selection. J Theor Biol 1966;12:12-45

15. Kaplan H. A theory of fertility and parental investment in traditional and modern human societies. Yearbook Phys Anthropol 1996;39:91135

16. Sear R, Mace R, McGregor IA. Maternal grandmothers improve nutritional status and survival of children in rural Gambia. Proc Roy Soc B 2000;267:1641-1647.

17. Packer C, Tatar M, Collins A. Reproductive cessation in female mammals. Nature 1998;392:807-811.

18. Grimes DA. The morbidity and mortality of pregnancy-still risky business. Am J Obst Gynecol 1994;170:1489-1494.

19. Abitbol MM, Chervenah FA, Ledger WJ. Birth and human evolution: anatomical and obstetrical mechanics in primates. Westport: Bergin and Garvey. 1996

20. O'Connell JF, Hawkes K, Blurton Jones NG. Grandmothering and the evolution of Homo erectus. J Hum Evol 1999;36:461-485.

21. Kirkwood TBL. Human senescence. Bioessays 1996;18:1009-1016.

22. Turke PW. Hypothesis: menopause discourages infanticide and encourages continued investment by agnates. Evol Hum Behav 1997;18: 3-13.

23. Finch CE. Longevity, senescence, and the genome. University of Chicago Press. 1990. 